\title{
Corrigendum to "Three-Dimensional Cobalt Hydroxide Hollow Cube/Vertical Nanosheets with High Desalination Capacity and Long-Term Performance Stability in Capacitive Deionization"
}

\author{
Yuecheng Xiong $\mathbb{D}^{1,2}$ Fei Yu, ${ }^{3}$ Stefanie Arnold $\mathbb{D},{ }^{4,5}$ Lei Wang $\mathbb{D},,^{4,5}$ Volker Presser $\mathbb{D}^{\mathbb{D}},{ }^{4,5,6}$ \\ Yifan Ren, ${ }^{1,2}$ and Jie Ma $\mathbb{D}^{1,2,7}$ \\ ${ }^{1}$ State Key Laboratory of Pollution Control and Resource Reuse, College of Environmental Science and Engineering, \\ Tongji University, Shanghai 200092, China \\ ${ }^{2}$ Research Center for Environmental Functional Materials, College of Environmental Science and Engineering, Tongji University, \\ 1239 Siping Road, Shanghai 200092, China \\ ${ }^{3}$ College of Marine Ecology and Environment, Shanghai Ocean University, Shanghai 201306, China \\ ${ }^{4}$ INM-Leibniz Institute for New Materials, 66123 Saarbrücken, Germany \\ ${ }^{5}$ Department of Materials Science and Engineering, Saarland University, 66123 Saarbrücken, Germany \\ ${ }^{6}$ Saarene-Saarland Center for Energy Materials and Sustainability, 66123 Saarbrücken, Germany \\ ${ }^{7}$ Shanghai Institute of Pollution Control and Ecological Security, Shanghai 200092, China \\ Correspondence should be addressed to Yuecheng Xiong; xiongyc@tongji.edu.cn
}

Received 3 November 2021; Accepted 3 November 2021; Published 1 December 2021

Copyright ( 2021 Yuecheng Xiong et al. Exclusive Licensee Science and Technology Review Publishing House. Distributed under a Creative Commons Attribution License (CC BY 4.0).

In the article titled "Three-Dimensional Cobalt Hydroxide Hollow Cube/Vertical Nanosheets with High Desalination Capacity and Long-Term Performance Stability in Capacitive Deionization" [1], there was an error in the title. It previously read "Three-Dimensional Cobalt Hydroxide Hollow Cube/Vertical Nanosheets with High Desalination Capacity and Long-Term Performance Stability", which was incorrect. The title in the original version has now been updated.

\section{References}

[1] Y. Xiong, F. Yu, S. Arnold et al., “Three-Dimensional Cobalt Hydroxide Hollow Cube/Vertical Nanosheets with High Desalination Capacity and Long-Term Performance Stability in Capacitive Deionization," Research, vol. 2021, article 9754145, 14 pages, 2021. 